Algebraic 83 Geometric $\mathcal{T}$ opology

Volume 5 (2005) 1585-1587

Published: 24 November 2005

ATG

\title{
The topological Hawaiian earring group does not embed in the inverse limit of free groups
}

\author{
PAUl FABel
}

\begin{abstract}
Endowed with natural topologies, the fundamental group of the Hawaiian earring continuously injects into the inverse limit of free groups. This note shows the injection fails to have a continuous inverse. Such a phenomenon was unexpected and appears to contradict results of another author.
\end{abstract}

AMS Classification 57M05, 14F35; 54H10, 22A10

Keywords Topological fundamental group, inverse limit space, Hawaiian earring

\section{Introduction}

Quite generally the based fundamental group $\pi_{1}(X, p)$ of a space $X$ becomes a topological group whose topology is invariant under the homotopy type of the underlying space $X$ (Corollary 3.4 [1]). In the context of spaces complicated on the small scale the utility of this invariant is emerging. For example topological $\pi_{1}$ has the potential to distinguish spaces when the algebraic homotopy groups fail to do so [2]. Unfortunately even in the simplest cases the topological properties of $\pi_{1}(X, p)$ can be challenging to understand.

Consider the familiar Hawaiian earring $X=\cup_{n=1}^{\infty} S_{n}$, (the union of a null sequence of simple closed curves $S_{n}$ joined at a common point) and the canonical homomorphism $\phi: \pi_{1}(X) \rightarrow \lim _{\leftarrow} \pi_{1}\left(\cup_{i=1}^{n} S_{i}\right)$.

The paper [1, page 370] seems to claim that $\phi$ is also a homeomorphism onto its image (" $\psi^{-1}$ is surely continuous as well..."). The intent of this note is to show that such a claim is false. The monomorphism $\phi$ is not a homeomorphism onto its image, and thus $\phi$ fails to be a topological embedding (Theorem 2.1). To prove this we consider the sequence $\left[\left(y_{1} * y_{n} * y_{1}^{-1} * y_{n}^{-1}\right)^{n}\right]$ where $y_{i}$ loops counterclockwise around the $i t h$ circle. The sequence diverges in $\pi_{1}(X, p)$ with the quotient topology but the sequence converges to the trivial element in the inverse limit space $\lim _{\leftarrow} \pi_{1}\left(\cup_{i=1}^{n} S_{i}\right)$. 


\section{Main Result}

Suppose $X$ is a topological space and $p \in X$. Endowed with the compact open topology, let $C_{p}(X)=\{f:[0,1] \rightarrow X$ such that $f$ is continuous and $f(0)=f(1)=p\}$. Then the topological fundamental group $\pi_{1}(X, p)$ is the quotient space of $C_{p}(X)$ obtained by treating the path components of $C_{p}(X)$ as points. Thus, letting $q: C_{p}([0,1], X) \rightarrow \pi_{1}(X, p)$ denote the canonical surjection, a set $A \subset \pi_{1}(X, p)$ is closed in $\pi_{1}(X, p)$ if and only if $q^{-1}(A)$ is closed in $C_{p}([0,1], X)$.

The space $Y$ is said to be $T_{1}$ if the one point subsets of $Y$ are closed.

If $A_{1}, A_{2}$, are topological spaces and $f_{n}: A_{n+1} \rightarrow A_{n}$ is a continuous surjection then, (endowing $A_{1} \times A_{2} \ldots$ with the product topology) the inverse limit space $\lim _{\leftarrow} A_{n}=\left\{\left(a_{1}, a_{2}, \ldots\right) \in\left(A_{1} \times A_{2} \ldots\right) \mid f_{n}\left(a_{n+1}\right)=a_{n}\right\}$.

The map $f:[0,1] \rightarrow Y$ is of the form $\alpha_{1} * \alpha_{2} \ldots * \alpha_{n}$ if there exists a partition $t_{0} \leq t_{1} \ldots \leq t_{n}$ of $[0,1]$ such that for each $i \geq 1$ we have $f_{\left[t_{i-1}, t_{i}\right]}=\alpha_{i}$.

For the remainder of the paper we use the following notation.

Let $X_{n}=\cup_{i=1}^{n}\left\{(x, y) \in R^{2} \mid\left(x-\frac{1}{n}\right)^{2}+y^{2}=\frac{1}{n^{2}}\right\}$. Note since $X_{n}$ is locally contractible the path components of $C_{p}\left(X_{n}\right)$ are open in $C_{p}\left(X_{n}\right)$ and hence the topological group $\pi_{1}\left(X_{n}, p\right)$ has the discrete topology.

Let $r_{n}^{*}: \pi_{1}\left(X_{n}, p\right) \rightarrow \pi_{1}\left(X_{n-1}, p\right)$ denote the epimorphism induced by the retraction $r_{n}: X_{n} \rightarrow X_{n-1}$ collapsing the $n^{\text {th }}$ circle to the point $p=(0,0)$. Let $\lim _{\leftarrow} \pi_{1}\left(X_{n}, p\right)$ denote the inverse limit space under the maps $r_{n}^{*}$.

Let $X=\cup_{n=1}^{\infty} X_{n}$ denote the familiar Hawaiian and let $R_{n}: X \rightarrow X_{n}$ denote the retraction fixing $X_{n}$ pointwise and collapsing $\cup_{i=n+1}^{\infty} X_{i}$ to the point $p$.

The formula $\phi([f])=\left(\left[R_{1}(f)\right],\left[R_{2}(f)\right], \ldots\right)$ determines a canonical homomorphism $\phi: \pi_{1}(X, p) \rightarrow \lim _{\leftarrow} \pi_{1}\left(X_{n}, p\right)$.

Remark The homomorphism $\phi: \pi_{1}(X, p) \rightarrow \lim _{\leftarrow} \pi_{1}\left(X_{n}, p\right)$ is continuous (Proposition 3.3 [1]) and one to one (Theorem 4.1 [3]). Since $\pi_{1}\left(X_{n}, p\right)$ is discrete the space $\Pi_{n=1}^{\infty} \pi_{1}\left(X_{n}, p\right)$ is metrizable and in particular the subspace $\lim _{\leftarrow} \pi_{1}\left(X_{n}, p\right)$ is a $T_{1}$ space. Consequently $\pi_{1}(X, p)$ is a $T_{1}$ space since $\phi$ is continuous and one to one. Thus the path components of $C_{p}(X)$ are closed in $C_{p}(X)$.

Theorem 2.1 The injection $\phi: \pi_{1}(X,\{p\}) \hookrightarrow \lim _{\leftarrow} \pi_{1}\left(X_{n}, p\right)$ is not a topological embedding. 
Proof Let $q=(2,0)$ in $X_{1}$. For a loop $f:[0,1] \rightarrow \cup_{i=1}^{\infty} X_{i}$ with base point $p=(0,0)$ define the oscillation number $O_{q}(f)$ as the maximal $n$ such that there exist $0=t_{0}<t_{1} \cdots t_{2 n-1}<t_{2 n}=1$ with $f\left(t_{2 i}\right)=p$ and $f\left(t_{2 i-1}\right)=q$. Let $y_{i} \in C_{p}(X)$ loop once counterclockwise around the $i$ th circle and let $y_{i}^{-1}$ $\in C_{p}(X)$ loop once clockwise around the ith circle.

First note that if $f \in C_{p}\left(\cup_{i=1}^{\infty} X_{i}\right)$ is path homotopic to a map of the form $\left(y_{1}^{-1} * y_{n}^{-1} * y_{1} * y_{n}\right)^{n}$ then $O_{q}(f) \geq 2 n$ for $n \geq 2$. To see this first observe $O_{q}(f)=O_{q}\left(R_{n} f\right)$. Now recall $\pi_{1}\left(X_{n}, p\right)$ is canonically isomorphic to the free group on generators $\left\{y_{1}, \ldots y_{n}\right\}$. Thus if $w$ is an (unreduced) word corresponding to $R_{n} f$ then each step of the algebraic reduction of $w$ to $\left(y_{1}^{-1} y_{n}^{-1} y_{1} y_{n}\right)^{n}$ never raises the oscillation number of the corresponding path in $X_{n}$. Hence $O_{q}(f) \geq$ $O_{q}\left(\left(y_{1}^{-1} * y_{n}^{-1} * y_{1} * y_{n}\right)^{n}\right)=2 n$.

To prove $\phi$ is not an embedding consider the set $A \subset \pi_{1}(X, p)$ defined as $\left.A=\left\{\left[f_{2}\right],\left[f_{3}\right] \ldots\right]\right\}$ where $f_{n}$ is of the form $\left(y_{1}^{-1} * y_{n}^{-1} * y_{1} * y_{n}\right)^{n}$. To see that $A$ is closed in $\pi_{1}(X, p)$ consider the union of (closed) path components $B=$ $\cup_{n=2}^{\infty}\left[f_{n}\right] \subset C_{p}(X)$. Observe if $f \in C_{p}(X)$ there exists an open neighborhood $U \subset C_{p}(X)$ such that $O_{q}(f) \geq O_{q}(g)$ for each $g \in U$. Thus $U \cap\left[f_{n}\right] \neq \emptyset$ for at most finitely many of the closed sets $\left[f_{n}\right]$. Hence $B$ is closed in $C_{p}(X)$ and consequently $A$ is closed in $\pi_{1}(X, p)$. On the other hand $\phi(A)$ is not closed in the image of $\phi$ since the sequence $\left\{\phi\left(\left[f_{n}\right]\right)\right\}$ converges to the trivial element in $\lim _{\leftarrow} \pi_{1}\left(X_{n}, p\right)$. Hence $\phi$ is not a homeomorphism from $\pi_{1}(X, p)$ onto the image of $\phi$.

\section{References}

[1] D K Biss, The topological fundamental group and generalized covering spaces, Topology Appl. 124 (2002) 355-371 MathReview

[2] P Fabel Topological fundamental groups can distinguish spaces with isomorphic homotopy groups, arXiv:math.AT/0502402

[3] J W Morgan, I Morrison, A van Kampen theorem for weak joins, Proc. London Math. Soc. (3) 53 (1986) 562-576 MathReview

Drawer MA, Department of Mathematics and Statistics

Mississippi State University, Mississippi State, MS 39762, USA

Email: fabel@ra.msstate.edu

URL: http://www2.msstate.edu/ fabel/

Received: 15 Febrary $2005 \quad$ Revised: 22 October 2005 